\title{
Unusual Fatal Infections after Anterior Cervical Spine Surgeries
}

\author{
Junseok W Hur ${ }^{1}$, Jang-Bo Lee ${ }^{2}$, Joo-Han Kim, Se-Hoon Kim ${ }^{3}$, Tai-Hyoung Cho ${ }^{2}$ \\ Jung-Keun Suh ${ }^{2}$, Youn-Kwan Park ${ }^{1}$ \\ Departments of Neurosurgery, ${ }^{1}$ Guro Hospital, ${ }^{2}$ Anam Hospital, ${ }^{3}$ Ansan Hospital, Korea University \\ College of Medicine, Seoul, Korea
}

We report two cases of cervical spinal epidural abscess (SEA), which are related to anterior cervical surgeries. The first case reveals a late postoperative infection without any predisposing factor. The second case reveals combined complication of infection and instrument failure (artificial disc). Both two cases manifested ascending infections that are unusual courses of anterior cervical infections. The abscess extended upwards and, finally, caused life threatening bacterial meningitis. We suggest aggressive surgical interventions with anti-bacterial therapies in such cases.

Key Words: Spinal epidural abscess $\cdot$ Cervical $\cdot$ Discectomy $\cdot$ Artificial disc replacement $\cdot$ Meningitis

\section{INTRODUCTION}

Anterior cervical discectomy with fusion (ACDF) is one of the most widely used techniques in the neurosurgical management of cervical spondylosis and disc herniation, ${ }^{3,8,11)}$. This procedure has very low complication rate; $0.1 \%$ to $1.6 \%{ }^{9}$. Most infections occur during the early postoperative period, as a result of operative seeding or poor wound care ${ }^{2)}$. But in case 1, spinal epidural abscess (SEA) occurred during the late postoperative period, and there was no evidence of esophageal perforation or Zenker's diverticulum.

There are some studies that address low instrument failure rate or infection rate after cervical artificial disc replacement (ADR), but a pool of evidence is still lacking ${ }^{4)}$. In case 2, we report ADR failure that was combined with SEA. Both cases reveal that SEA may cause ascending infection, extending to upper cervical and finally to brain, resulting in life threatening septic condition due to bacterial meningitis.

\section{CASE REPORT}

\section{Case 1}

A healthy 68-year-old man underwent surgery from another

- Received: July 29, 2012 • Revised: September 20, 2012

- Accepted: September 25, 2012

Corresponding Author: Jang-Bo Lee, MD, PhD

Department of Neurosurgery, Korea University Anam Hospital, 73, Inchon-ro, Seongbuk-gu, Seoul 136-705, Korea

Tel: +82-2-920-5729, Fax: +82-2-929-0629

Email: jblee42@gmail.com clinic for C5-6 and C6-7 disc herniation. The surgery was performed by the anterior cervical approach consisting of discectomy followed by fusion (ACDF) with allograft and platescrew construction. The postsurgical period was uneventful.

14 months later, the patient was transferred to our hospital with progressive paraparesis (upper extremities motor grade $3 / 2$, lower extremities motor grade $3 / 3$ ), urinary retention and posterior neck pain that started 2 weeks before. He had no signs of fever or superficial wound infection. X-ray and Computer Tomography (CT) demonstrated moderate graft extrusion and screw loosening. But most important finding was gas collection on ventral portion of epidural space (Fig. 1A). Gadolinium-enhanced magnetic resonance imaging (enhanced MRI) was performed directly to reveal high-signal intensity SEA with a diameter of $10 \mathrm{~mm}$. The SEA was located in the anterior epidural space from $\mathrm{C} 4$ to $\mathrm{C} 5$, and caused marked compression of the spinal cord (Fig. 1B). On enhanced T1-weighted axial cut, additionally to SEA, abscess pockets were also observed on both lateral sides of plate (Fig. 1C).

There was no sign of superficial wound infection, including fever. There was no definite clinical evidence of comorbid infection. But, laboratory infection markers were high. Estimated sedimentation rate (ESR) was $35 \mathrm{~mm} / \mathrm{hr}$ (normal range 0-15 $\mathrm{mm} / \mathrm{hr}$ ), C-reactive protein (CRP) was $193.4 \mathrm{mg} / \mathrm{L}$ (normal range 0-3 mg/L), and the white blood cell (WBC) count was 16,100/ $\mathrm{mm}^{3}$ (normal range $4,500-11,000 / \mathrm{mm}^{3}$ ).

Immediate decompressive surgery was performed. Pus was noted on C4-5 prevertebral space where metal plate was placed. We removed all artificial materials including allograft bone. C4 and C5 corpectomy was performed. Greenyellowish pus was extracted from anterior epidural space. Vertebra body 

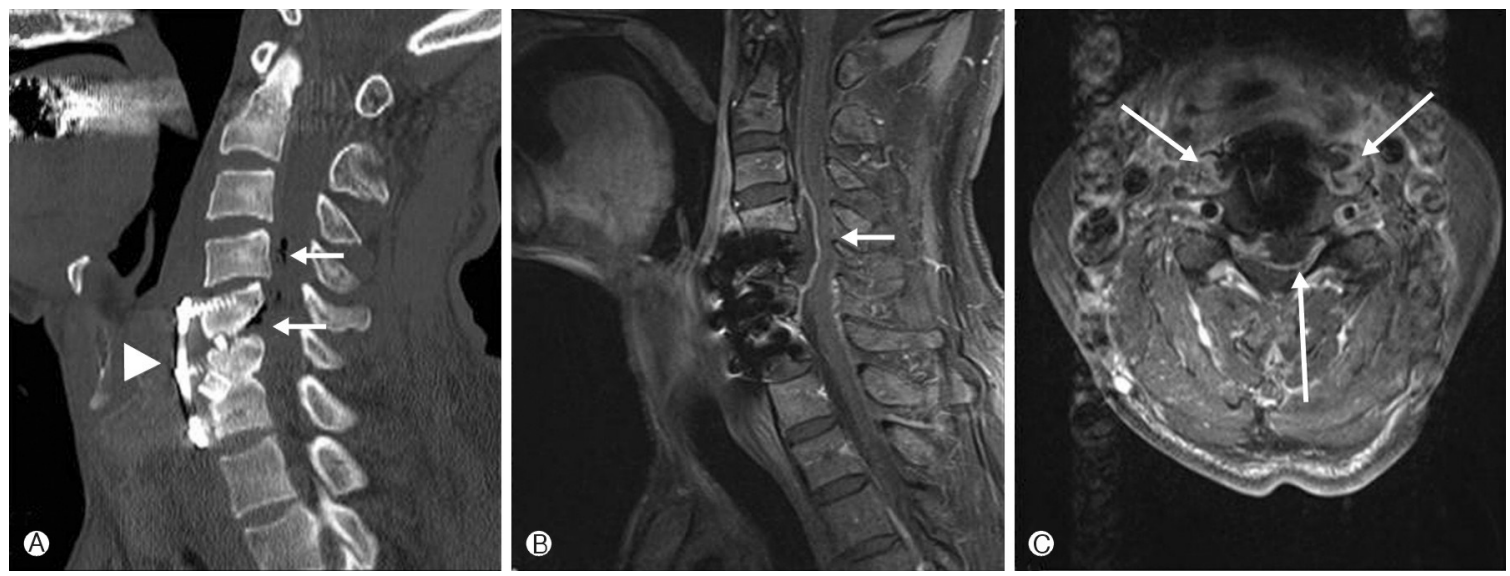

Fig. 1. Sagittal CT of the cervical spine demonstrates moderate graft extrusion, screw loosening (arrow head), and gas collection on ventral portion of epidural space (arrows) (A). Sagittal MRI demonstrates epidural abscess leading to marked compression of the spinal cord (arrow) (B). Axial MRI shows epidural abscess and peri-instruments abscess (arrows) (C).
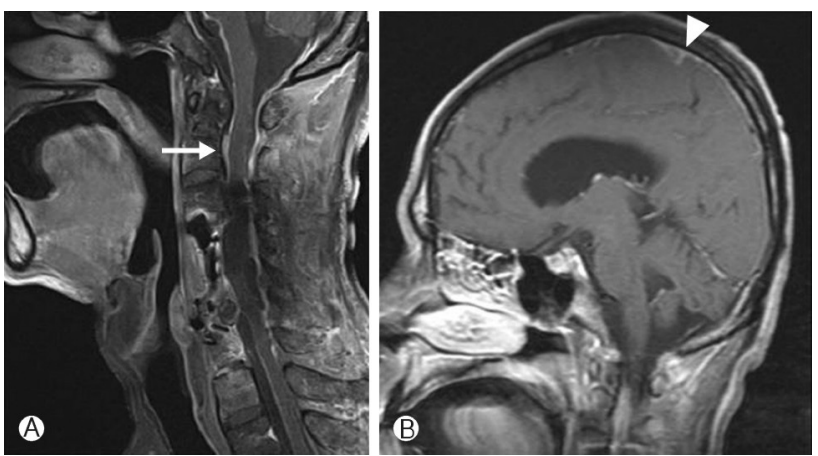

Fig. 2. Sagittal MRI shows epidural abscess ascended to the $C 2$ ventral level and (arrow) ( $A$ ) and meningeal enhancement (arrow head) (B).

and pus were sent for microscopic culture and sensitivity examination. Epidural space was massively irrigated with normal saline. Before closure, esophagus was inspected, and esophagography was performed after surgery. There was no evidence of an esophageal injury.

Empirically, Vancomycin was administered immediately after surgery to cover methicillin-resistant Staphylococcus aureus $(\mathrm{MRSA})^{5,17}$. But tissue culture revealed Streptococcus anginosus so the patient was treated with appropriate $2^{\text {nd }}$ line antibiotics (Ceftriaxone + Metronidazole). We performed an additional surgery by posterior approach on postoperative day 9 for stabilization. Posterior midline approach with pedicle screw fixation (Vertex; Medtronic, Inc., Minneapolis, MN, USA) and interlaminar fusion was performed on C3-7. After surgery, there was an immediate neurological recovery - up to upper extremities motor grade $4 / 3$, lower grade extremities motor $4 / 4$. Vital signs were stable and laboratory studies showed improvement of infectious condition. But in week 3, quadriparesis was aggravated. Enhance MRI showed an epidural abscess that as- cended to the C2 ventral level (Fig. 2A). Re-exposure of previous anterior surgical site and massive irrigation was held. Greenyellowish pus was still noted and culture biopsy was performed.

The pathogens isolated were MRSA, and Klebsiella pneumonia. Therefore, the anti-microbial therapy was changed to Vancomycin and Meropenem.

The patient was continued on antibiotics for 7 weeks, but anterior cervical wound was still unhealed and pus leaked out. We explored the wound and daily irrigation was held under local anesthesia. Antibiotics were maintained. 4 weeks after wound exploration, the patient showed altered mentality. Enhanced brain MRI showed meningeal enhancement (Fig. 2B) and cerebrospinal fluid (CSF) analysis showed an elevated white cell count of $870 / \mathrm{mm}^{3}$ (normal range $0 / \mathrm{mm}^{3}$ ), and protein concentration of $86 \mathrm{mg} / \mathrm{dL}$ (normal range $10-45 \mathrm{mg} / \mathrm{dL}$ ). Bacterial meningitis was strongly suspected. As $3^{\text {rd }}$ line antibiotic therapy seemed to have failed, Linezolid was started for $4^{\text {th }}$ line. After 4 months, patient expired due to progressive sepsis.

\section{Case 2}

A 52-year-old male presented with a C6-7 disc herniation resulting in right arm radicular pain. Physical examination demonstrated no apparent abnormal finding. He underwent an uncomplicated C6-7 discectomy and artificial disc replacement (Prodisc-C; Synthes, West Chester, PA, USA) (Fig. 3A). After surgery, right arm pain improved, and he was discharged home on postoperative day 4 without any event.

2 weeks later, the patient represented with sudden neck pain. X-ray examination demonstrated instrument displacement and subsidence (Fig. 3B). We planned for revision the next day, and routine lab for operation preparation was held. ESR/CRP were elevated to $60 \mathrm{~mm} / \mathrm{h} / 66.7 \mathrm{mg} / \mathrm{L}$ but he was in afebrile state 

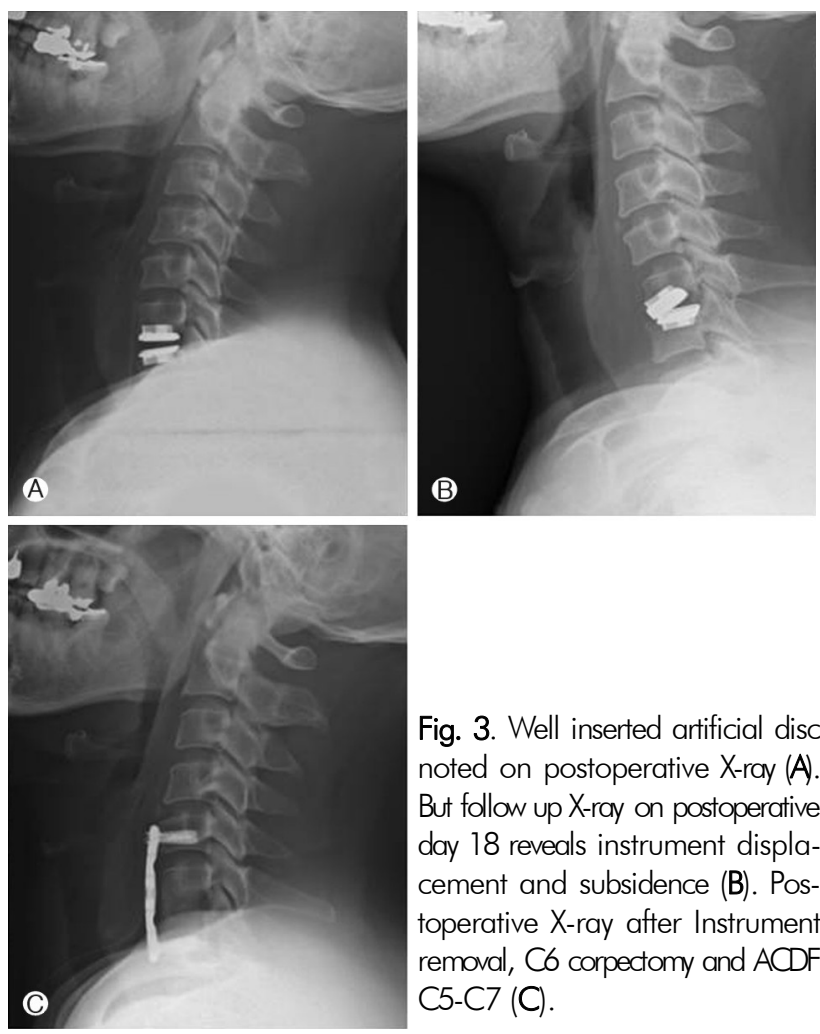

Fig. 3. Well inserted artificial disc noted on postoperative X-ray (A). But follow up X-ray on postoperative day 18 reveals instrument displacement and subsidence (B). Postoperative $\mathrm{X}$-ray after Instrument removal, $\mathrm{C} 6$ corpectomy and $\mathrm{ACDF}$ C5-C7 (C).

with no clinical evidence to suspect infection besides neck pain.

Upon exploration of previous operation site, small amount of yellowish pus like discharge was found at prevertebral space just anterior to $\mathrm{C} 6$ and artificial disc. Culture biopsy was taken. The instrument was removed, followed by massive irrigation. C6 vertebra body showed some inflammation reaction, and therefore, corpectomy was performed. Bone graft, harvested from the iliac crest, was placed between the vertebral bodies $\mathrm{C} 5 / \mathrm{C} 7$, and an anterior plate was applied (Vectra; Synthes, West Chester, PA, USA) (Fig. 3C). Vancomycin was started empirically and MRSA was isolated from the pus. But WBC, ESR, CRP were rising, wound was unhealed, pus leakage persisted, and fever did not subside. Antibiotic treatment was changed to Teicoplanin on postoperative day 7. Enhanced MRI was performed on postoperative day 14. Epidural abscess was noted on C3-7, resulting in slight compression of the cord (Fig. 4A). Emergency surgery was performed. We removed the implant and massive irrigation was held. But 7 days after surgery, patient showed sudden weakness on right upper extremity to grade 1. Enhanced MRI was performed immediately. Epidural abscess showed aggravation, ascending extension from C7 to C2 level (Fig. 4B). Another emergency surgery was performed. At first, C2-6 corpectomy was performed via previous incision. The abscess was debrided, irrigated and evacuated. Specimens were sent for culture. The pathogen isolated was
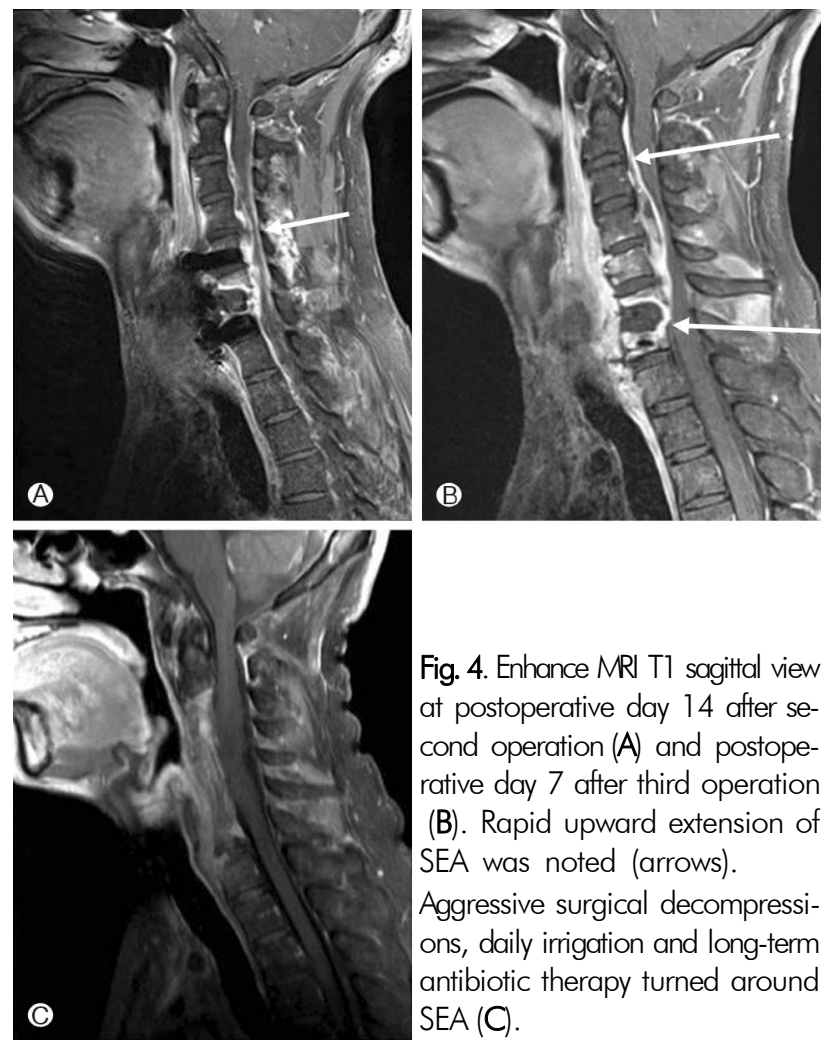

Fig. 4. Enhance MRI T1 sagittal view at postoperative day 14 after second operation (A) and postoperative day 7 after third operation (B). Rapid upward extension of SEA was noted (arrows).

Aggressive surgical decompressions, daily irrigation and long-term antibiotic therapy turned around SEA (C).

also MRSA, so that Teicoplanin was maintained. Wound was unclosed and daily irrigation was performed. Few days later, altered mentality and unstable vital sign were noted, and the symptoms continued for more than a week. Patient was treated in intensive care unit because of bacterial meningitis and septic condition. 3 weeks after, infection seemed to be under control. Mentality and vital sign improved. ESR/CRP dropped continuously till 44/13.35 and there was no more discharge from wound. So wound was closed under general anesthesia. After another 4 weeks of absolute bed rest, we permitted walkerassisted ambulation with four poster brace wearing. Antibiotics were maintained for additional 2 weeks. 2 months later, ESR/ CRP declined to 14/0.7 and follow up MRI revealed complete resolution of the epidural abscess and no further evidence of spinal cord compression (Fig 4C). Therefore, we attempt a last surgery for stabilization. The initial plan was anterior and posterior fusion, but anterior surgical plane was too adhesive and dural thinning was noted, indicating that anterior fusion had failed. In the posterior fusion approach, polyaxial screw-rod system (Synapes; Synthes, West Chester, PA, USA) was used for C2-T2 fixation through the pedicle (Fig. 5). A strong framework was required for stability due to anterior fusion failure.

The patient's neurology improved - right upper motor up to grade 4 , and other motor grades 5 , and laboratory tests 

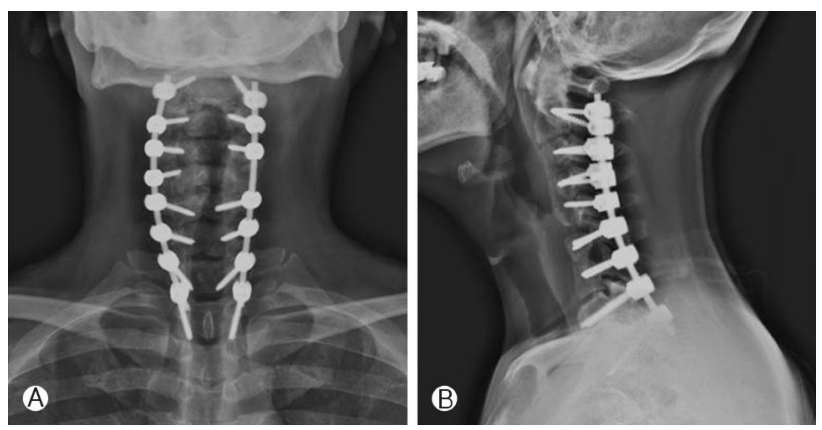

Fig. 5. Pedicle screw insertion from C2 to T2.

also returned to normal. He was discharged and there was no additional event during outpatient follow-up period.

\section{DISCUSSION}

ACDF and ADR are commonly performed to treat cervical disc herniation ${ }^{3,8)}$. Postoperative spine infections are rare due to the rich blood supply of the vertebrae and the prophylactic use of antibiotics ${ }^{12)}$. However, the use of instrumentation exhibit a higher risk of infection ${ }^{13)}$. The pathogenetic mechanism of postoperative SEA is assumed to be a result of direct inoculation of the offending pathogen during surgery, and/or dissemination from other infection sites ${ }^{18)}$.

In general, infections associated with ACDF occur in the early postoperative period. If they occur late after surgery, esophageal perforation or Zenker's diverticulum should be suspected ${ }^{16)}$. Many patients have one or more predisposing factors such as underlying disease, previous intervention, or a potential local or systemic cause of infection ${ }^{6}$. Case 1 is a unique example of a SEA in the absence of, esophageal perforation, diverticulum, and other previously reported risk factors for late infection. Only few cases were reported in this condition. The presence of spinal implants may be a risk factor for deep, delayed wound infection in the setting of surgery. Micro injury of esophagus due to implants or microtrauma effect must be strongly considered in pathophysiology ${ }^{2)}$.

ADR associated infection rate and implant failure rate are both low $^{4}$. In case 2, both complications occurred concomitantly, and such cases have not been reported well previously.

SEA, as postoperative infection, shows rapid and lifethreatening progression. Early detection and aggressive treatments including surgical intervention and antibiotics are important ${ }^{1,5)}$. But in real field, it is not easy to suspect SEA, and if diagnosed after severe neurologic deficit, its morbidity and mortality are high, ${ }^{5,17}$. In our cases, both patients showed ascending extension of SEA that led to bacterial meningitis and septic conditions.
Fever, back pain, and progressive neurologic deficits have been thought as the triad of spinal epidural abscess ${ }^{1,17}$. The CRP level should also be determined in suspected or confirmed cases of SEA. The CRP level rises faster at the onset of inflammation and returns to normal sooner than the $\mathrm{ESR}^{19)}$.

Staphylococcus aureus has been reported as the most frequently causative organism for spinal epidural abscess ${ }^{5,17}$. Therefore, before pathogen confirmation, prophylactic antibacterial therapy should cover a broad spectrum that includes Staphylococcus aureus.

Enhanced MRI is the imaging method of choice because it is highly sensitive, less invasive, delineates both the longitudinal and paraspinal extensions of the abscess, and may help differentiate infections from cancer on the basis of the appearance and the signal intensity of the image ${ }^{15)}$.

The management of SEA has remained controversial, with both proponents of non-operative and of operative treatments reporting good results. Several authors have shown satisfactory results in patients treated non-operatively ${ }^{14)}$. However, nonoperative managements require longer protracted courses of antibiotics and close neurological monitoring ${ }^{7}$. If neurological abnormalities occur, there is no doubt in performing surgical interventions in early period ${ }^{1)}$. A surgery is performed with the aim of obtaining a definitive pathological and microbiological diagnosis, followed by decompression of neural tissue. Posterior longitudinal ligament (PLL) should be eliminated because PLL and epidural venous plexus are important routes of ascending infection ${ }^{10)}$.

\section{CONCLUSION}

Anterior cervical surgery related infections are rare. But once such a case of deep infection as SEA occurs, aggressive surgical intervention should be considered. These two cases presented here reveal atypical ascending infections. We assume aggressive surgical intervention with anti-bacterial treatments are necessary for prevention of life-threatening courses in such cases.

\section{REFERENCES}

1. Baker AS, Ojemann RG, Swartz MN, Richardson EP, Jr.: Spinal epidural abscess. N Engl J Med 293:463-468, 1975

2. Christiano LD, Goldstein IM: Late prevertebral abscess after anterior cervical fusion. Spine (Phila Pa 1976) 36:E798-802, 2011

3. Cloward RB: The anterior approach for removal of ruptured cervical disks. J Neurosurg 15:602-617, 1958

4. Coric D, Nunley PD, Guyer RD, Musante D, Carmody CN, Gordon CR, et al: Prospective, randomized, multicenter study of cervical arthroplasty: 269 patients from the kineflexc artificial 
disc investigational device exemption study with a minimum 2-year follow-up: clinical article. J Neurosurg Spine 15:348-358, 2011

5. Curry WT Jr., Hoh BL, Amin-Hanjani S, Eskandar EN: Spinal epidural abscess: clinical presentation, management, and outcome. Surg Neurol 63:364-371; discussion 371, 2005

6. Darouiche RO: Spinal epidural abscess. N Engl J Med 355: 2012-2020, 2006

7. Del Curling O Jr., Gower DJ, McWhorter JM: Changing concepts in spinal epidural abscess: a report of 29 cases. Neurosurgery 27:185-192, 1990

8. Espersen JO, Buhl M, Eriksen EF, Fode K, Klaerke A, Kroyer L, et al: Treatment of cervical disc disease using cloward's technique. i. general results, effect of different operative methods and complications in 1,106 patients. Acta Neurochir (Wien) 70:97114, 1984

9. Fountas KN, Kapsalaki EZ, Nikolakakos LG, Smisson HF, Johnston KW, Grigorian AA, et al: Anterior cervical discectomy and fusion associated complications. Spine (Phila Pa 1976) 32:23102317, 2007

10. Jevtic V: Vertebral infection. Eur Radiol 14 Suppl 3:E43-52, 2004

11. Kim SH, Kim SD, Keem SH, Park JY, Lim DJ, Cho TH, et al: Clinical experience with solis ${ }^{\mathrm{TM}}$ cage for anterior cervical interbody fusion in degenerative cervical diseases: a preliminary report. Korean J Spine 1:192-200, 2004

12. Kulkarni AG, Hee HT: Adjacent level discitis after anterior cer- vical discectomy and fusion (acdf): a case report. Eur Spine J 15 Suppl 5:559-563, 2006

13. Levi AD, Dickman CA, Sonntag VK: Management of postoperative infections after spinal instrumentation. J Neurosurg 86: 975-980, 1997

14. Leys D, Lesoin F, Viaud C, Pasquier F, Rousseaux M, Jomin $M$, et al: Decreased morbidity from acute bacterial spinal epidural abscesses using computed tomography and nonsurgical treatment in selected patients. Ann Neurol 17:350-355, 1985

15. Parkinson JF, Sekhon LH: Spinal epidural abscess: appearance on magnetic resonance imaging as a guide to surgical management. report of five cases. Neurosurg Focus 17:E12, 2004

16. Pompili A, Canitano S, Caroli F, Caterino M, Crecco M, Raus L, et al: Asymptomatic esophageal perforation caused by late screw migration after anterior cervical plating: report of a case and review of relevant literature. Spine (Phila Pa 1976) 27: E499-502, 2002

17. Rigamonti D, Liem L, Sampath P, Knoller N, Namaguchi Y, Schreibman DL, et al: Spinal epidural abscess: contemporary trends in etiology, evaluation, and management. Surg Neurol 52:189-196; discussion 197, 1999

18. Silber JS, Anderson DG, Vaccaro AR, Anderson PA, Mc-Cormick P: Management of postprocedural discitis. Spine J 2:279287, 2002

19. Sorensen P: Spinal epidural abscesses: conservative treatment for selected subgroups of patients. Br J Neurosurg 17:513-518, 2003 